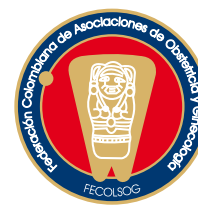

EDITORIAL

\title{
LOS EVENTOS ADVERSOS EN LA ATENCIÓN EN SALUD
}

\section{Adverse events arising from medical management}

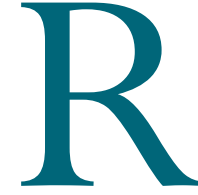

ecientemente ha recibido mucha atención el tema de los eventos adversos, tanto a nivel local como internacional. Declaraciones de quienes dirigen los organismos multilaterales y nacionales de salud pública respecto a la magnitud del problema y su impacto, ${ }^{1}$ el desarrollo de iniciativas de financiación para su estudio y búsqueda de soluciones. ${ }^{2}$ Publicación de artículos periodísticos que presentan los diferentes puntos de vista de los pacientes, las instituciones y los entes que controlan la calidad de los servicios de salud..$^{3-5}$ Pronunciamientos de las aseguradoras respecto a la intención de no pagar las cuentas de los pacientes que hayan presentado ciertos eventos adversos. ${ }^{6}$ Realización del seminario internacional de Seguridad del Paciente, llevado a cabo este mes en Bogotá, y difusión de los resultados de investigaciones científicas realizados en el país acerca de la frecuencia y evitabilidad de los mismos, la cual recibió el Premio de la Academia Nacional de Medicina en Ciencias Clínicas. ${ }^{7}$ Éstos son algunos ejemplos de la extensión en que se trata el tópico en la actualidad.

El tema genera gran controversia y posiciones antagónicas que surgen por varios motivos: definiciones deficientes o incompletas, diferentes aproximaciones al error humano, que son aplicadas desde disímiles puntos de vista, algunas veces en defensa de intereses particulares. Variadas metodologías de detección, evaluación, análisis e investigación, que requieren mayor valoración para conocer su validez y utilidad en diferentes escenarios culturales, y por último, diferentes respuestas de las sociedades contemporáneas para afrontarlos.

Respecto a la definición, vale la pena aclarar que como evento adverso se califica a las situaciones que terminan en daño no intencional al paciente, como consecuencia del cuidado provenido o con ocasión de éste, más que como consecuencia de la enfermedad de base. ${ }^{8}$ Un ejemplo de esta situación es la lesión de la vejiga en una cirugía por endometriosis severa. Más allá de lo indicada que pudiera estar la cirugía, si ésta no se hubiera realizado, no habría ocurrido la lesión de la vejiga; de este modo algunos eventos adversos serán no prevenibles. Otro ejemplo sería una infección de la herida en un cesárea de urgencia en la que no se aplicó oportunamente el antibiótico profiláctico, posiblemente esta infección hubiera podido prevenirse.

El problema con las definiciones proviene de la definición de error médico, la cual proviene del término "Medical Errors". Según el término MeSH, acuñado en 1997 por la Biblioteca Nacional de Medicina de los Estados Unidos, se define como: los errores cometidos por los profesionales de la salud, que resultan en daño al paciente. ${ }^{9}$ Esta definición está de acuerdo al abordaje individual del error humano. Esta limitada definición nos conduce al segundo punto en conflicto, sobre cómo se debe abordar el error humano.

Las teorías más antiguas abogan por el abordaje individual del error humano, consideran que se debe centrar la atención del error en las personas que están al final del acto inseguro; sea el médico, la enfermera o el farmaceuta. El error surge como consecuencia de un proceso mental aberrante, como la negligencia, el desinterés y la falta de atención, los cuales son propios del ser humano. Los seguidores de esta aproximación manejan los errores bajo conceptos morales: "las cosas malas les pasan a las malas personas”, los psicólogos lo llaman la hipótesis del mundo justo. ${ }^{10}$ Como lógica 
consecuencia, las medidas para corregirlos deben ir dirigidas a corregir este mal comportamiento, entonces se debe buscar un culpable para avergonzarlo y castigarlo como medida ejemplarizante para que no vuelvan a suceder. Esta aproximación es frecuentemente utilizada en las instituciones de salud, por parte de algunos gerentes, que mediante la búsqueda del culpable, intentan minimizar la responsabilidad institucional ante posibles demandas civiles o glosas en las cuentas por parte de las aseguradoras. También por parte de la sociedad, representada por el pensamiento de la prensa, que se ejemplifica en el editorial del periódico El Tiempo del día 26 de octubre titulado: "Cuando el médico falla", en el que sentencia: "Al parecer, la justicia está decidida a meter en cintura a los profesionales sanitarios...". ${ }^{11}$

Por otra parte, está la aproximación sistémica al error humano, en estrecha relación con conceptos gerenciales modernos, como el pensamiento sistémico propuesto por Senge en $1990^{12}$ para ser aplicado en el error en medicina en al año 2000 por el psicólogo inglés James Rason. ${ }^{13}$ Según este abordaje, el error humano se puede presentar aún en las mejores organizaciones, y dado que la naturaleza humana no se puede cambiar, se requiere modificar las condiciones en las que se desempeñan los seres humanos, para que ser reduzca el riesgo de error. Este es el abordaje que se utiliza en otras industrias como la aviación o la energía nuclear, en las que se pretende minimizar el riesgo de error, mediante la creación de barreras que lo prevengan. El sujeto es más bien una victima de la falta de defensas del sistema. Tiene la gran ventaja de permitírsele aprender de los errores.

En cuanto a la metodología para su estudio, hay aproximaciones que abogan por el autoreporte y la vigilancia activa durante la estancia en el hospital, otras abogan por razones de eficiencia y para que la vigilancia sea posterior a la salida del paciente. Otros plantean la vigilancia pasiva mediante estudios de corte transversal. Otra discusión gira alrededor de si se deben estudiar sólo los eventos que produjeron daño para evitar su repetición, o si también se deben incluir los eventos que podrían haber terminado en daño; sin embargo, si no hay una oportuna evaluación o análisis que conduzca a la toma de medidas administrativas correctivas, ambos abordajes podrían ser contraproducentes.

Por último, respecto a cómo las sociedades pretenden enfrentar el problema, están quienes abogan por el modelo americano de demandas e indemnizaciones que conllevan a mayores $\operatorname{costos},{ }^{14}$ y producir mayor distorsión de las relaciones que deben regir un sistema de salud entre las entidades reguladoras, las aseguradoras, las instituciones de salud, los trabajadores y los pacientes.

Otros respaldan el modelo adoptado por las sociedades más avanzadas en la provisión de servicios de salud, y en especial en el manejo del error, que han concluido que las demandas médicas sólo logran encarecer los costos de los relacionados. Por ejemplo, en Nueva Zelanda ha llegado el sistema de "No Fallas", en el cual no se permite la instauración de demandas contra el personal o las instituciones de salud. El estado asume los costos derivados de los malos desenlaces procedidos de la provisión del cuidado o con ocasión de éste, por medio con un seguro similar al SOAT aquí en Colombia. Al mismo tiempo, entidades encargadas de vigilar e investigar las acciones del personal de salud o de las instituciones, se encargan de tomar los correctivos necesarios que haya a lugar. ${ }^{15}$

El tema es de gran interés para nuestra especialidad, ya que potencialmente puede afectar a la madre, al feto o al recién nacido. La frecuencia de eventos adversos en obstetricia es baja, comparada con otras especialidades; ${ }^{16,17}$ sin embargo la evitabilidad de los eventos es mayor. ${ }^{8}$ Además de los vacíos del conocimiento ya mencionados, existen algunos específicos a nuestro quehacer que requieren pronto estudio: se debe determinar la sensibilidad y especificidad de los criterios de detección utilizados de manera genérica en los servicios quirúrgicos y médicos ${ }^{18}$ así como la posible asociación entre el no cumplimento de los criterios de calidad de atención de la paciente obstétrica y la aparición de eventos adversos, también los costos relacionados, y por último, pero no menos importante, cómo involucrar 
a los pacientes y sus familiares en la resolución del evento. Debemos los obstetras y ginecólogos, los médicos familiares, los médicos generales y las enfermeras que brindan atención materna perinatal, trabajar conjuntamente con las instituciones de salud y los pacientes en los aspectos de la seguridad; ya sea su prevención, detección, evaluación y análisis, para llevar a intervenciones que reduzcan el riesgo de error en nuestros procesos de atención y en programas de mejoramiento de calidad, que hagan más seguras nuestras instituciones y nuestro ejercicio médico, y nos dejen menos vulnerables a los apetitos de abogados y aseguradoras interesadas más en su propio beneficio, que en el de los pacientes y la sociedad en general.

\section{Hernando Gaitán D.}

Editor

\section{REFERENCIAS}

1. World Health Organization. World Alliance for Patient Safety.Visitado en 2008 Nov 28. Disponible en http:// www.who.int/patientsafety/en/

2. World Health Organization. World Alliance for Patient Safety. Small Research Grants. Visitado en 2008 Nov 28. Disponible en: http://www.who.int/patientsafety/ research/grants/Smallgrants_summary_A4English.pdf

3. Evento adverso: error que cuesta. Errores médicos los paga el paciente. UN Periódico. 117. Visitado en 2008 Nov 28. Disponible en: http://unperiodico.unal.edu. co/ediciones/117/11.html

4. El médico la segunda víctima. UN Periódico. 117 Visitado en 2008 Nov 28. Disponible en: http:// unperiodico.unal.edu.co/ediciones/117/14a.html

5. Tasa de eventos adversos cercana al 10\%. UN Periódico. 117. Visitado en 2008 Nov 28. Disponible en: http:// unperiodico.unal.edu.co/ediciones/117/11.html.

6. Rosenthal MB. Nonpayment for performance? Medicare's new reimbursement rule. N Engl J Med 2007;357:1573-5.
7. Gaitán-Duarte H, Eslava-Schmalbach J, RodríguezMalagón N, Forero-Supelano V, Santofimio-Sierra $\mathrm{D}$, Altahona $\mathrm{H}$, et al. Incidencia y evitabilidad de eventos adversos en pacientes hospitalizados en tres instituciones hospitalarias en Colombia, 2006. Rev salud pública 2008;10:215-26.

8. Reportable Events Guidelines. Ministry of Health New Zeland, 2001. Visitado en 2005 Jun 10. Disponible en: http://www.moh.govt.nz Ministry of health New Zealand.

9. MeSh Home - Pubmed/MEDLINE. National Library of Medicine. Visitado en 2008 Nov 28. Disponible en: http://www.ncbi.nlm.nih.gov/sites/entrez? db=mesh

10. Lerner MJ. The desire for justice and reactions to victims. En: McCauley J, Berkowitz L, eds. Altruism and helping behavior. New York: Academic Press;1970.

11. Cuando el médico falla. El Tiempo, Editorial del 26 de Octubre de 2008.

12. Senge P. La Quinta Disciplina. El arte y la práctica de la organización abierta al aprendizaje. Barcelona: Granica Editores; 1992.

13. Reason J. Human error: models and management. BMJ 2000;320:768-70.

14. Studdert DM, Mello MM, Gawande AA, Gandhi TK, Kachalia A, Yoon C, et al. Claims, errors, and compensation payments in medical malpractice litigation. N Engl J Med 2006;354:2024-33.

15. Davis P, Lay-Yee R, Fitzjohn J, Hider P, Briant R, Schug S. Compensation for medical injury in New Zealand: does "'no-fault" increase the level of claims making and reduce social and clinical selectivity? J Health Polit Policy Law 2002;27:833-54.

16. Vincent C, Neale G, Woloshynowych M. Adverse events in British hospitals: preliminary retrospective record review. BMJ 2001;322:517-9.

17. Michel P, Quenon JL, de Sarasqueta AM, Scemama O. Comparison of three methods for estimating rates of adverse events and rates of preventable adverse events in acute care hospitals. BMJ 2004;328:199-203.

18. Baker GR, Norton PG, Flintoft V, Blais R, Brown A, Cox J, et al. The Canadian Adverse Events Study: the incidence of adverse events among hospital patients in Canada.CMAJ 2004;170:1678-86. 